\title{
Prevalence of Depression in Patients with Diabetic Retinopathy
}

\author{
Nooshin Bazzazi ${ }^{1}$ iD , Mona Sharafi Zamir ${ }^{2}$, Siamak Akbarzadeh $^{1}$, Younes Mohammadi ${ }^{3}$, \\ Mohammad Ahmadpanah,"* \\ ${ }^{1}$ Associate Professor, Department of Ophthalmology, School of Medicine, Hamadan University of Medical Sciences, \\ Hamadan, Iran \\ ${ }^{2}$ General Practitioner, School of Medicine, Hamadan University of Medical Sciences, Hamadan, Iran \\ ${ }^{3}$ Associate Professor, Department of Epidemiology, School of Health, Hamadan University of Medical Sciences, Hamadan, \\ Iran \\ ${ }^{4}$ Professor of Cognitive and Clinical Psychology, Research Center for Behavioral Disorders and Substance Abuse, Hamadan \\ University of Medical Sciences, Hamadan, Iran
}

* Corresponding Author: Mohammad Ahmadpanah, Research Center for Behavioral Disorders and Substance Abuse, Hamadan University of Medical Sciences, Hamadan, Iran. Email: m1ahmad2000@ gmail.com

Received: 03.11.2020 Accepted: 28.01 .2021

How to Cite this Article: Bazzazi N, Sharafi Zamir M, Akbarzadeh S, Mohammadi Y, Ahmadpanah M. Prevalence of Depression in Patients with Diabetic Retinopathy. Avicenna J Clin Med. 2021; 27(4): 226-231. DOI: $10.29252 /$ ajcm.27.4.226

\section{Abstract}

Background and Objective: Diabetic retinopathy is one of the most important and debilitating complications of diabetes with an increasing prevalence in most countries. It has a dramatic effect on people's quality of life and increases the symptoms of anxiety and depression in patients with diabetes. Therefore, the present study aimed to determine the prevalence of depression in patients with diabetic retinopathy.

Materials and Methods: This descriptive/ cross-sectional study was conducted on 245 patients with retinopathy referred to the ophthalmology clinic of Farshchian Hospital in Hamadan during 2019- 2020. They were selected via available and consecutive sampling method and were evaluated for depression with the 21-item Beck questionnaire. Data were analyzed in SPSS software (version 16) at a 95\% confidence level.

Results: The mean scores of patients' age and duration of diabetes were reported as $54.47 \pm 8.5$ and $8.5 \pm 4.15$ years, respectively. In terms of gender, the majority of participants $(58.7 \%)$ were female. The prevalence of depression in patients with diabetic retinopathy was obtained at $49.8 \%$ and in proliferative and non-proliferative retinopathy were $68.2 \%$ and $43 \%$, respectively $(\mathrm{P}<0.001)$. The prevalence rates of depression in men and women were reported as $45.9 \%$ and $51.5 \%(\mathrm{P}=0.428)$. Moreover, in patients treated with insulin and without insulin, the prevalence rates of depression were obtained at $50.4 \%$ and $49.2 \%(\mathrm{P}=0.846)$. The correlation coefficients of depressive disorder with age and duration of diabetes were calculated at $0.123(\mathrm{P}=0.054)$ and $0.369(\mathrm{P}<0.001)$, respectively.

Conclusion: Depression is one of the most serious problems in patients with diabetic retinopathy. As evidenced by the results of the present study, the prevalence of depression is significantly correlated with the severity of retinopathy and duration of diabetes.

Keywords: Depression, Diabetes Mellitus, Retinopathy 


\section{بررسى شيوع افسردَى در بيماران مبتلابه رتينوياتى ديابتى}

(iD)

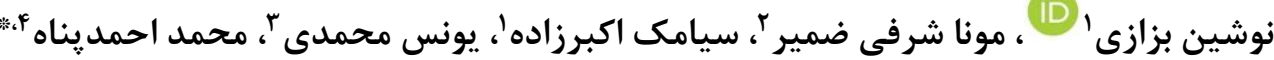

' دانشيار، كروه جشمريزشكى، دانشكده يزشكى، دانشكاه علوم يزشكى همدان، همدان، ايران

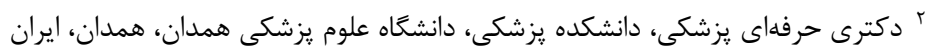

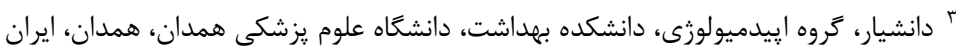

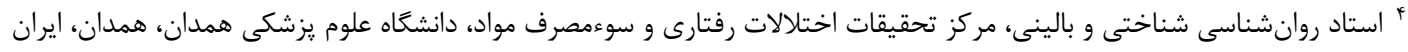
* نويسنده مسئول: محمد احمديناه، مركز تحقيقات اختلالات رفتارى و سوءمصرف مواد، دانشعاه علوم يزشكى همدان، همدان، ايران. ايميل: m1ahmad2000@gmail.com

\begin{tabular}{|c|c|}
\hline 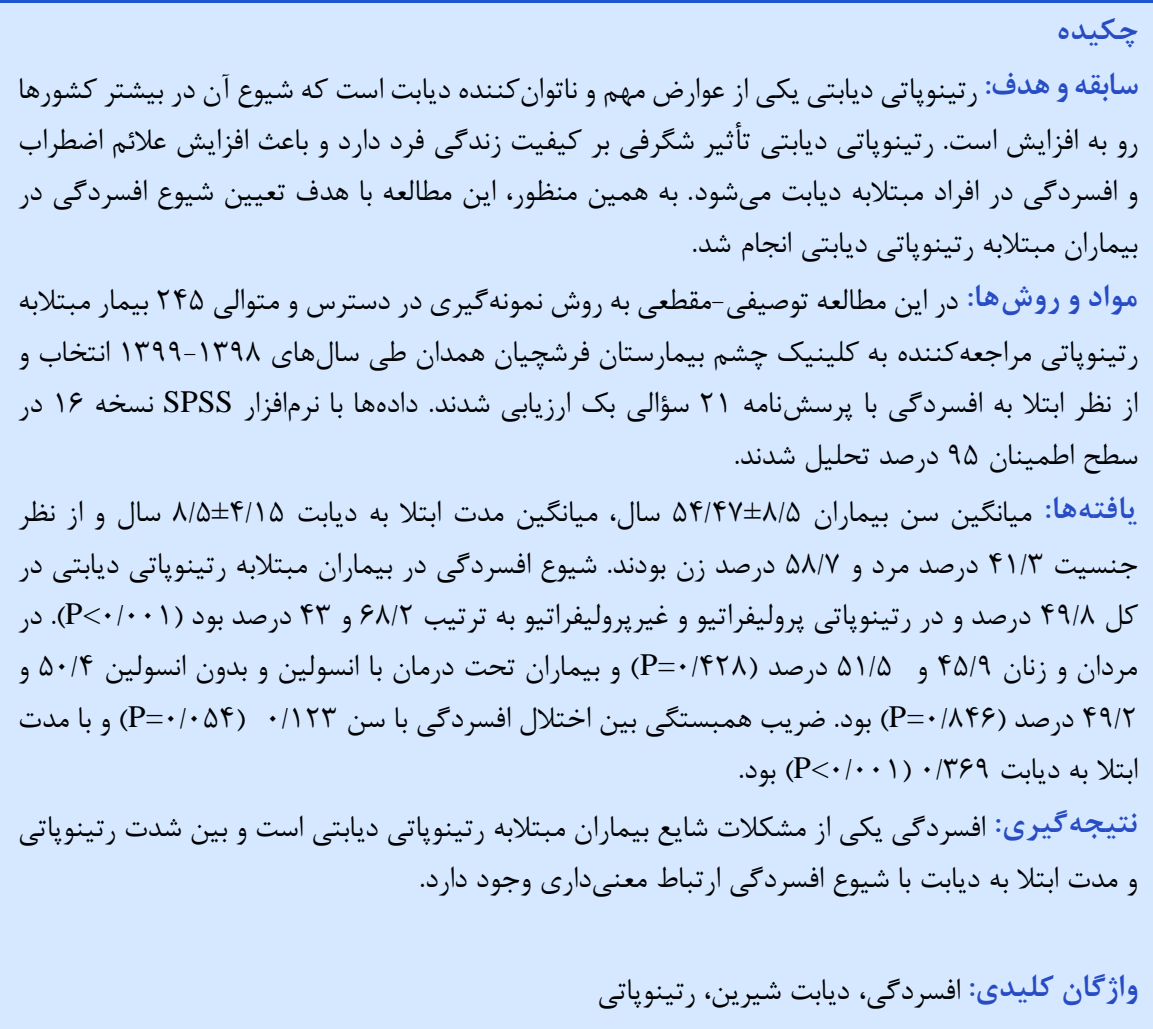 & 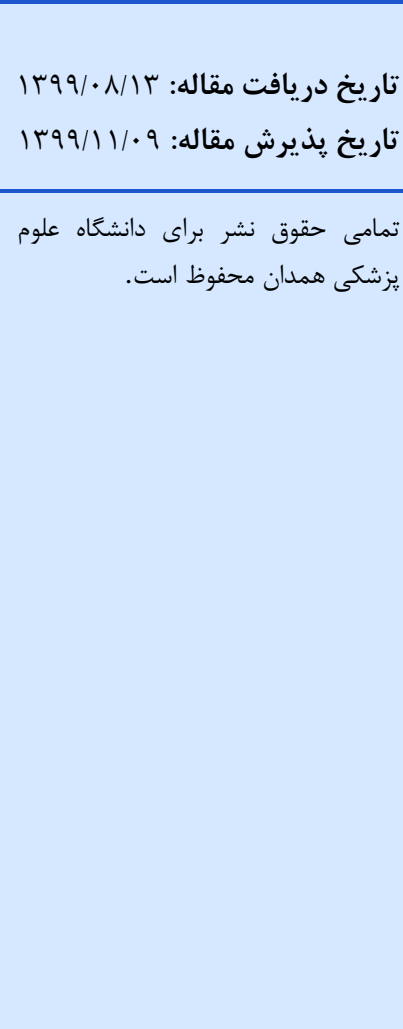 \\
\hline
\end{tabular}

رتينوياتى ديابتى و ادم ماكولاى ديابتيك دو علت مهم كاهش

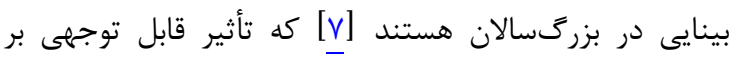

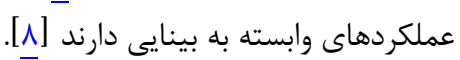

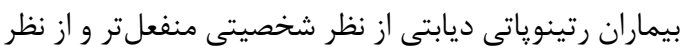

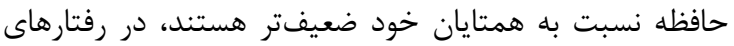

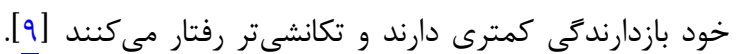

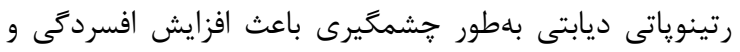

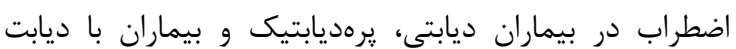

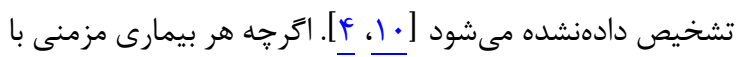

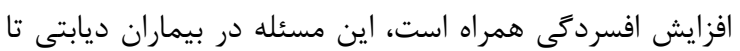

ديابت قندى از شايعترين بيمارىهاى متابوليك است [1]].

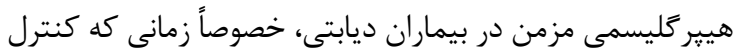

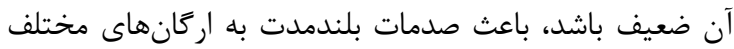

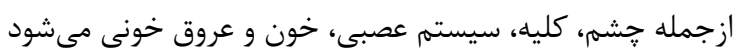

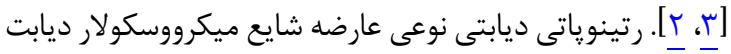

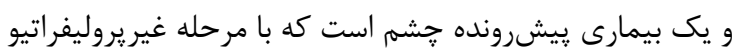

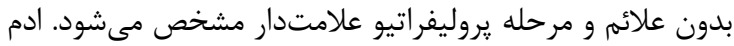

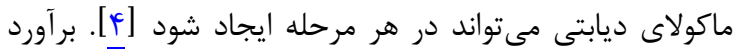

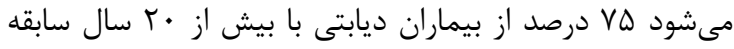

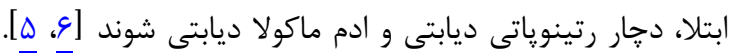


آزمون منويتنى در سطح اطمينان ه9 درصد تحليل شدند.

\section{إفته ماف}

در كل TFA بيمار ديابتى مبتلابه رتينوياتى بررسى شدند.

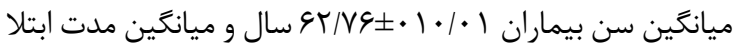

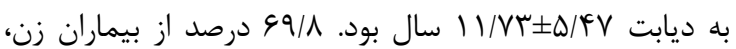

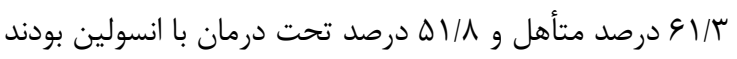

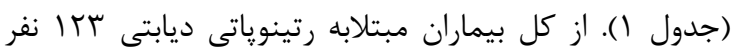

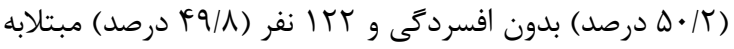

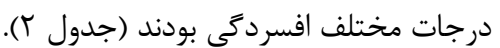

جدول ا: توزيع فراوانى اطلاعات پايه بيماران مبتلابه رتينوياتى ديابتى مطالعهشده

\begin{tabular}{|c|c|c|}
\hline درصد & تعداد & \\
\hline & & جنسيت \\
\hline$r \cdot / r$ & VF & 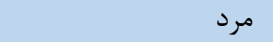 \\
\hline \multirow[t]{2}{*}{$99 / 1$} & $|V|$ & زن ان \\
\hline & & وضعيت تأهل \\
\hline $91 / \pi$ & $10 \cdot$ & همسردار \\
\hline$r V / l$ & 91 & همسر مرده \\
\hline$\cdot 1 \mathrm{~A}$ & r & همسر جداشده \\
\hline \multirow[t]{2}{*}{$\cdot / \Lambda$} & r & هرَز ازدواجنكرده \\
\hline & & سابقه بيمارى جسمى \\
\hline$\wedge 9 / \wedge$ & rT. & بلى بلى \\
\hline \multirow[t]{2}{*}{$1 \cdot / r$} & ra & خير \\
\hline & & تحت درمان با انسولين \\
\hline$\Delta) / \Lambda$ & ITV & بلى بلى \\
\hline YN/T & 111 & خير \\
\hline
\end{tabular}

ميانگين سن بيماران مبتلا و غيرمبتلا به افسردگى به ترتيب

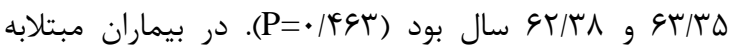

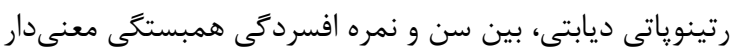

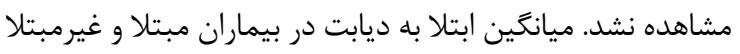

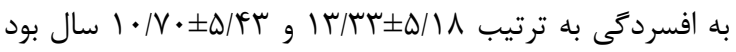

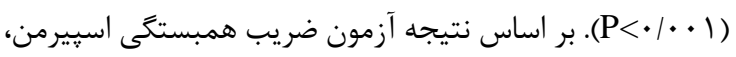

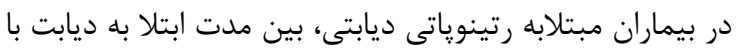

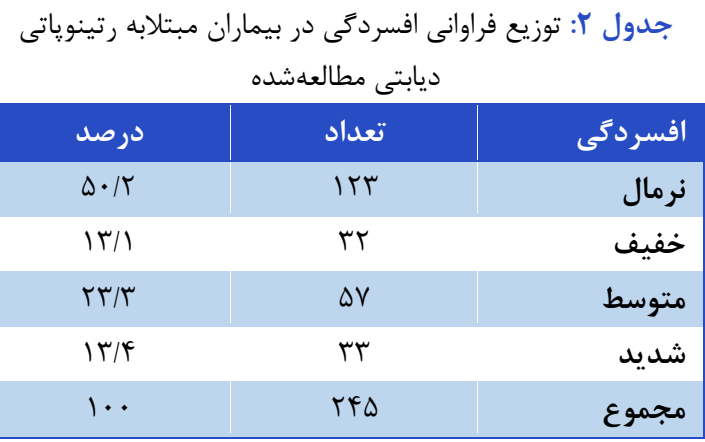

سه برابر شايعتر است [11]]. وجود افسردىى و اضطراب در

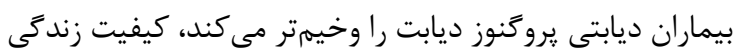

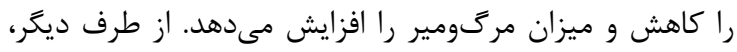

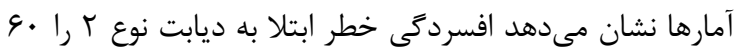

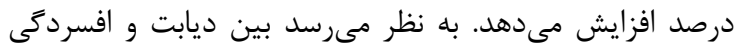

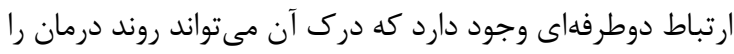

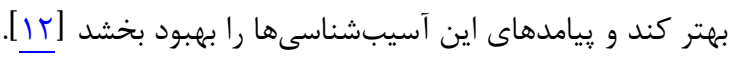

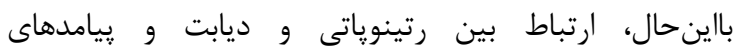

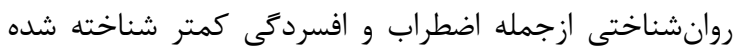

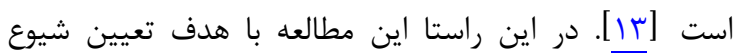
افسردكى در بيماران مبتلابه رتينوياتى ديابتى انجام شد.

\section{مواد و روشها}

مطالعه حاضر از نوع توصيفى است كه به روش مقطعى روى روى

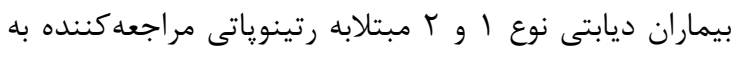

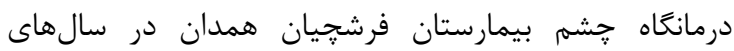

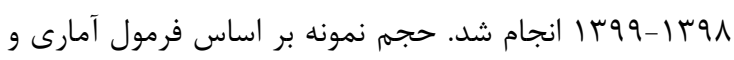

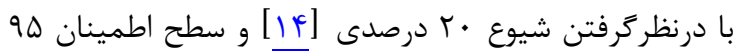

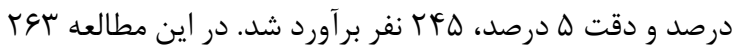

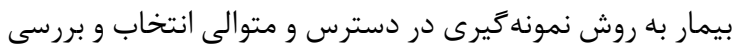

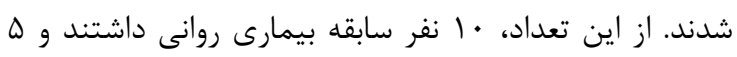

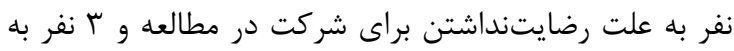
علت كاتاراكت شديد و عدم امكان معاينه شبكيه از مطالعه كنار كذاشته شدند. خصوصيات دموكر افيك افراد مطالعهشده شامل سن، جنس، تحصيلات، وضعيت تأهل، سوابق افسردگى، نوع ديابت و شدت

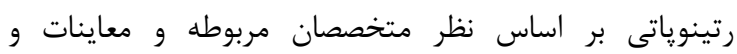

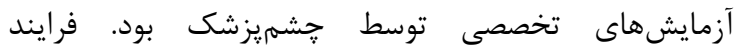
نمونه

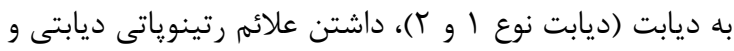

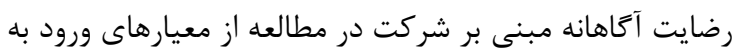

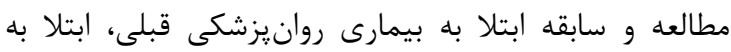
اختلالات بينايى غيرديابتيك و ديابت تأييدنشده از معيارهاى

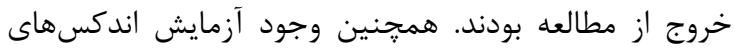

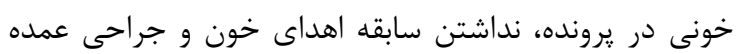
سه ماه قبل از مطالعه از معيارهاى ورود به مطالعه و ابتلا به به ندانه

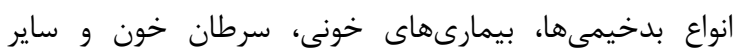

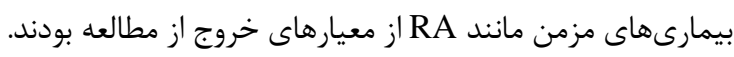

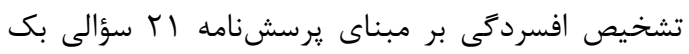

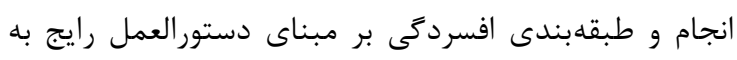

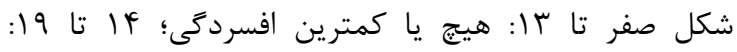

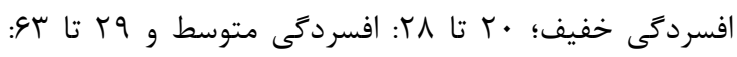

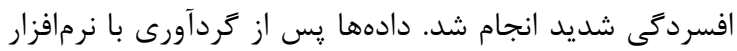

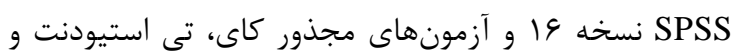


جدول ب: توزيع فراوانى اختلال افسردگى در بيماران مبتلابه رتينوياتى ديابتى مطالعهشده برحسب جنس، مصرف انسولين، بيمارى جسمى همراه، شدت رتينوياتى و وضعيت تأهل

\begin{tabular}{|c|c|c|c|c|}
\hline \multirow[b]{2}{*}{ ارزش P" } & \multicolumn{3}{|c|}{ اختلال افسردكى } & \\
\hline & تعداد (درصد) مجموع) & 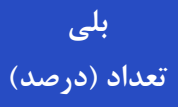 & تعداد (درصد) خير & \\
\hline - IFTA & $\begin{array}{l}(1 \cdots) V F \\
(1 \cdots) \mid V I\end{array}$ & $\begin{array}{l}(F \Delta / Q) \mathcal{F}^{F} \\
(\Delta / / \Delta) \wedge \Lambda\end{array}$ & $\begin{array}{l}(\Delta F / I) F . \\
(\mid \mathcal{F} / \Delta) \wedge r\end{array}$ & جنسيت \\
\hline - ANFE & $\begin{array}{l}(1 \cdots) \mid T V \\
(1 \cdots) 111\end{array}$ & $\begin{array}{l}(\Delta \cdot / F) G F \\
(F q / Y) \Delta \Lambda\end{array}$ & $\begin{array}{l}(\uparrow q / \varepsilon) \xi \mu \\
(\Delta \cdot / \Lambda) q .\end{array}$ & خلى مصرف انسولين \\
\hline.$/ 4 q$. & $\begin{array}{l}(1 \cdots) r r . \\
(1 \cdots) r \Delta\end{array}$ & $\begin{array}{l}(\Delta \cdot / 9) \| 1 T \\
(F \cdot / \cdot) \| .\end{array}$ & $\begin{array}{l}(\xi q / 1)) \cdot 1 \\
(\xi \cdot / \cdot)) \Delta\end{array}$ & بيمارى جسمى همراه \\
\hline$<\cdot / \cdot \cdot 1$ & $\begin{array}{l}(1 \cdots) 99 \\
(1 \cdots) 1 \vee 9\end{array}$ & $\begin{array}{l}(r \mid / \Lambda) Y I \\
(\Delta V / \cdot)) \cdot r\end{array}$ & 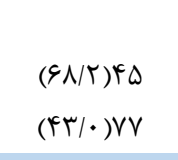 & 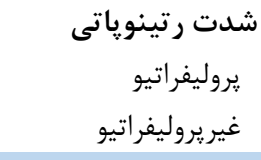 \\
\hline$\cdot 1 \cdot p q$ & $\begin{array}{l}(1 \cdots) 1 \Delta \cdot \\
(1 \cdots) 9 \Delta\end{array}$ & $\begin{array}{l}(\Delta \Delta / r) \wedge r \\
(\mathcal{E} / /) \mathcal{F} .\end{array}$ & $\begin{array}{l}(Y F / V) \& V \\
(\Delta V / q) \Delta \Delta\end{array}$ & وضعيت تأهل \\
\hline
\end{tabular}

ديابتى ه9 درصد، در مطالعه Bao و همكاران [IV] در در آمريكادر

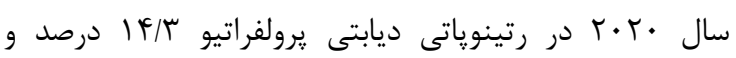

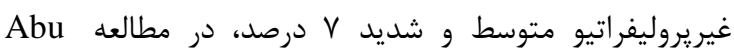

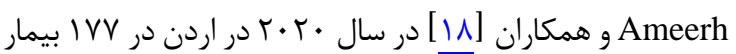

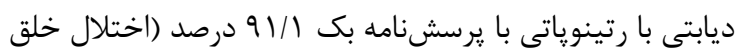

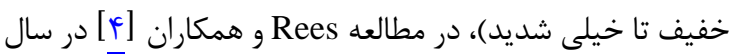

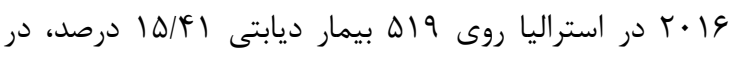

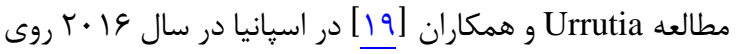

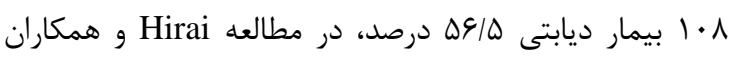

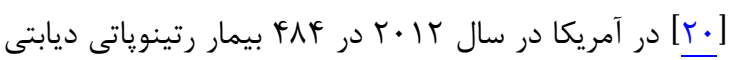

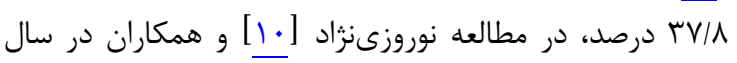

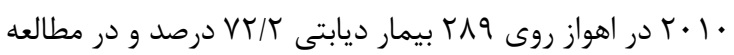

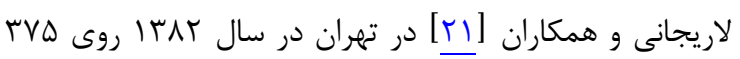

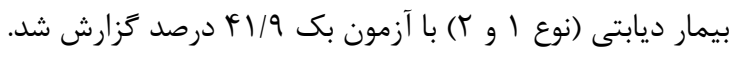

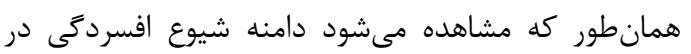

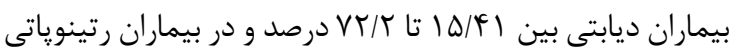

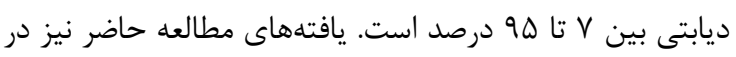

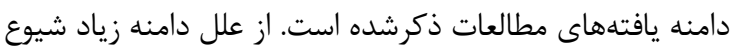

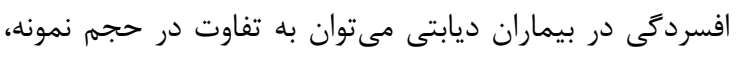

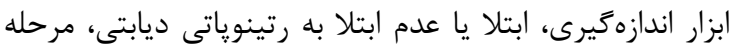

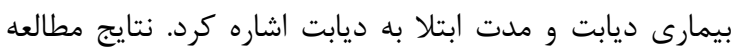

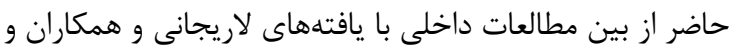

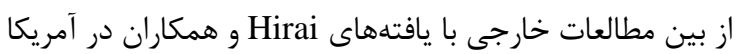

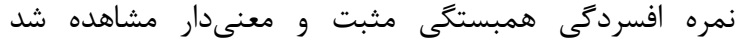

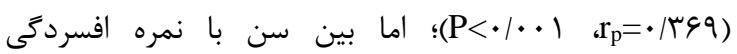

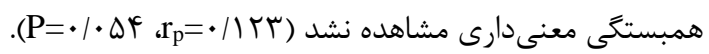

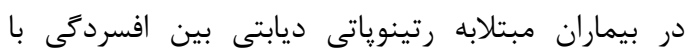

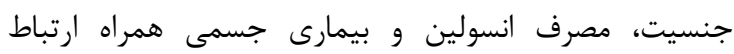
معنى دار مشاهده نشد. فراوانى اختلال افسردكى در درى بيماران

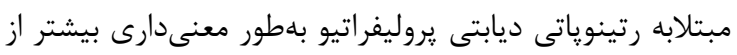

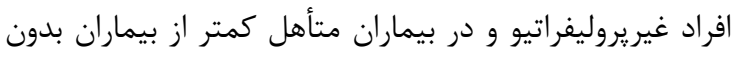
همسر (همسر مرده، همسر جداشده، هركز ازدواجنكرده) بود

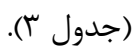

در مطالعه حاضر شيوع افسردگى در بيماران مبتلابه

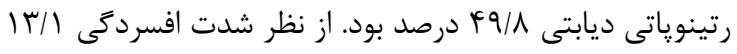

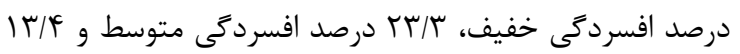

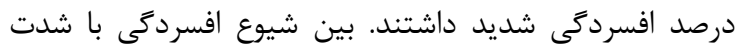

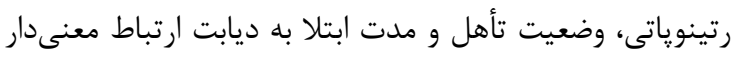

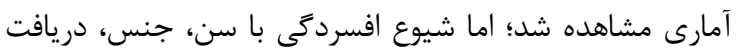
انسولين و سابقه بيمارى جسمى ارتباط معنى دار نداشت.

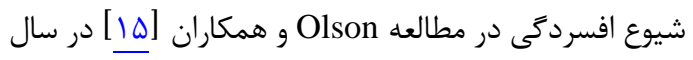

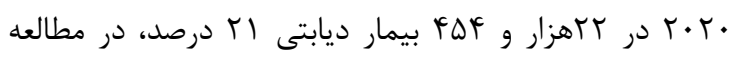
Ahmed

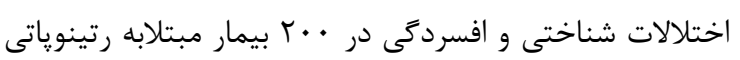


همكاران بين علائم افسردىى و متغيرهاى سن، جنس و عوارض دار دار

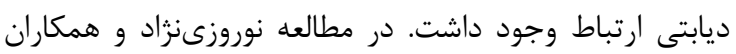

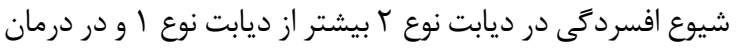
خوراكى بيشتر از تزريق انسولين بود.

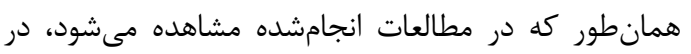

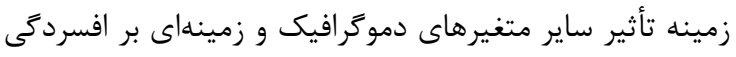

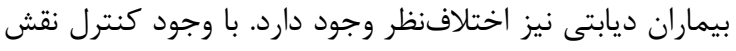

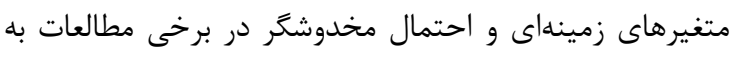

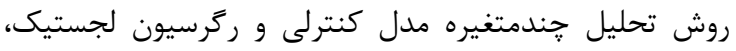

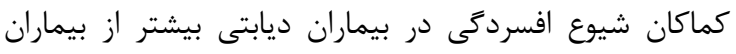

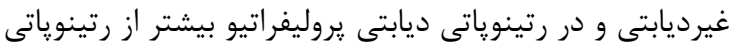

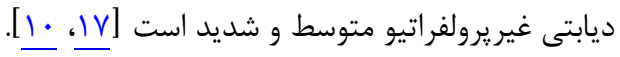
تعيين همزمان شدت رتينوياتى در بيماران ديابتيك توسط

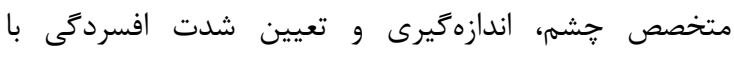

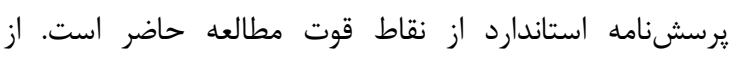

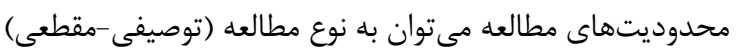

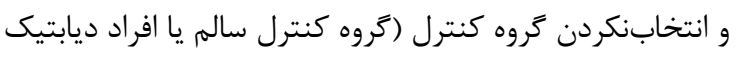

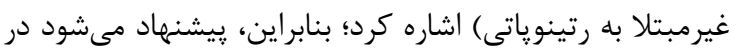

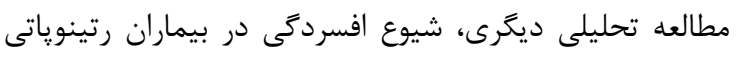

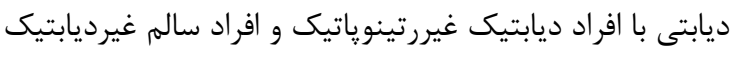

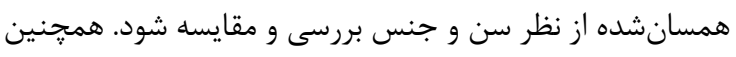

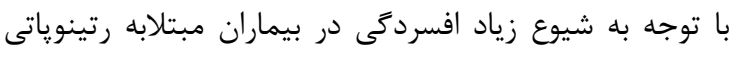

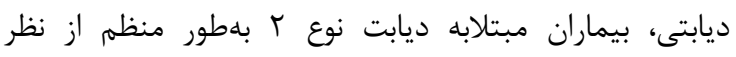

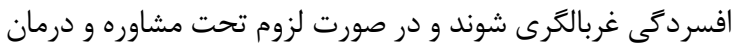

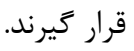

\section{نتيجه تيرى}

يافتههاى اين مطالعه نشان داد افسردى يكى يكى از مشكلات

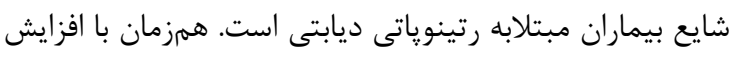

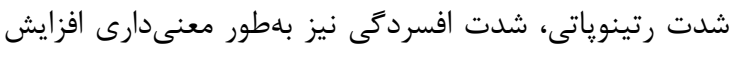

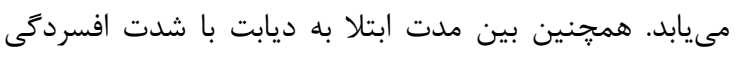
همبستخى مثبت و معنى دارى وجود دارد.

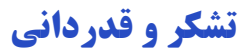

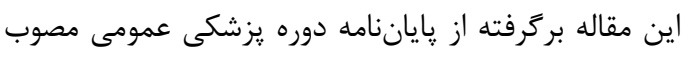

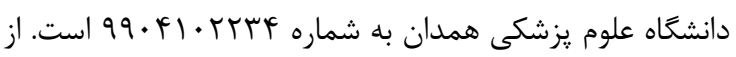

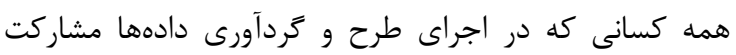
داشتندتقدير و تشكر مى كنيه.

تضاد منافع

نتايج اين مطالعه با منافع نويسندكان در تعارض نيست.

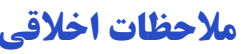

اين يروزه از كميته اخلاق دانشكاه علوم يزشكى همدان با

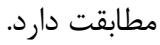

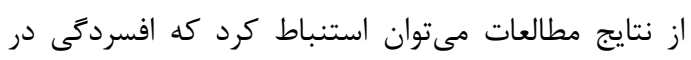

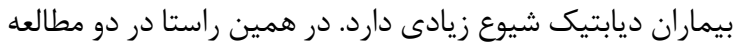

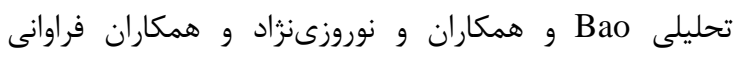

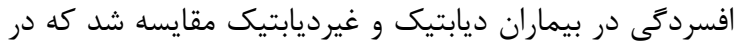

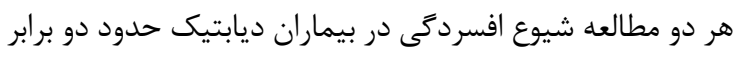

بيمار ان غيرديابتيك بود. سؤالى كه در اينجا مطرح مىشود ايني اين است كه آيا بين

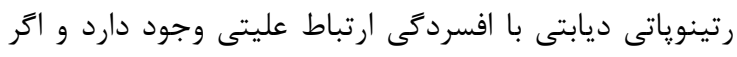

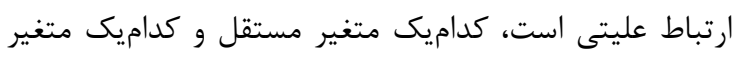

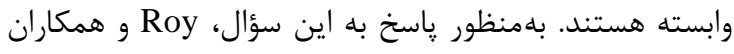

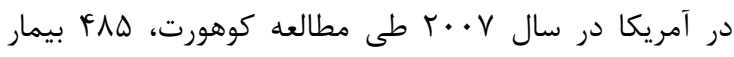

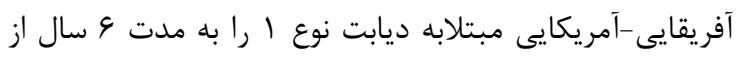

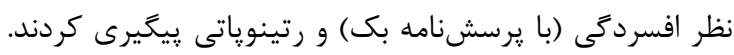

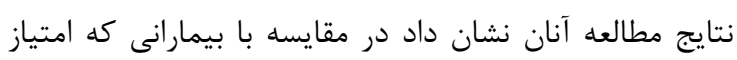

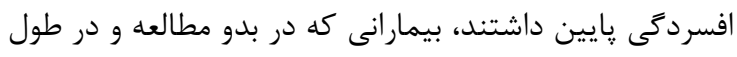

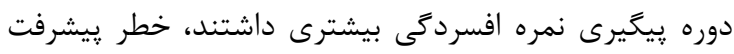

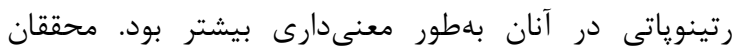

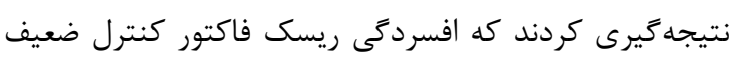

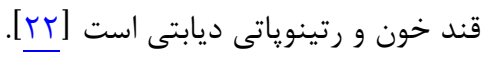

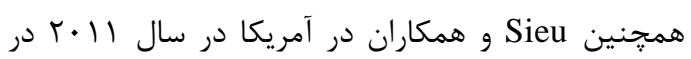

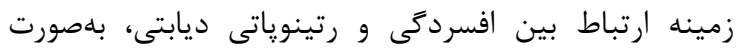
كوهورت كذشتهنكر و ز

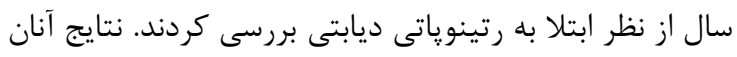

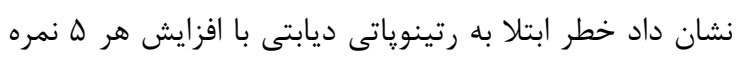

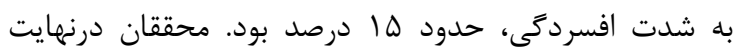

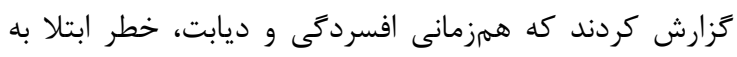

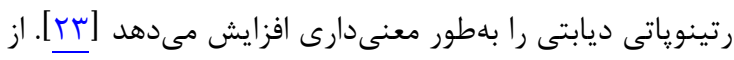

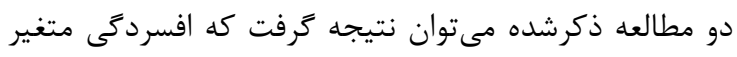

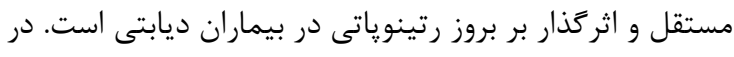

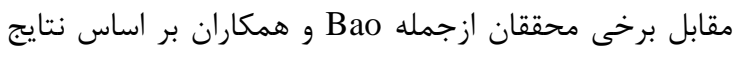

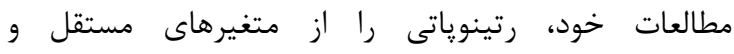

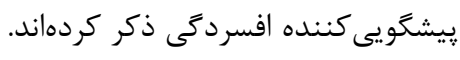

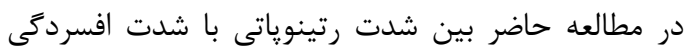

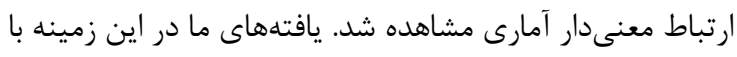

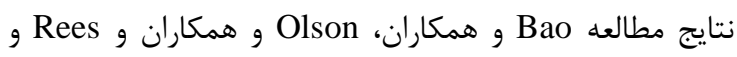

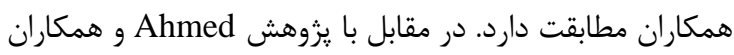

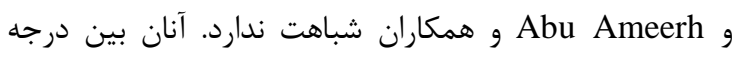

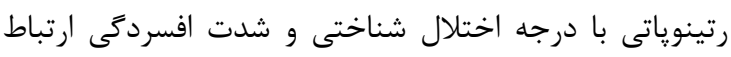

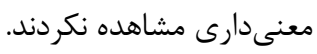

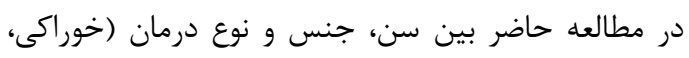

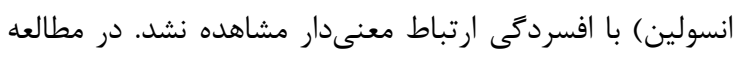

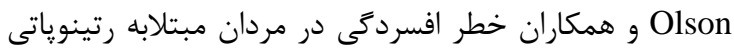

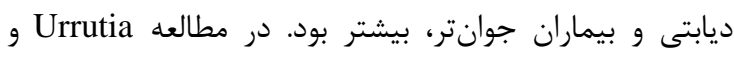




$$
\begin{aligned}
& \text { (يزوهشگر همكار): تدوين بخش روششناسى و تحليل آمارى }
\end{aligned}
$$

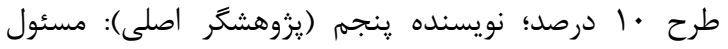

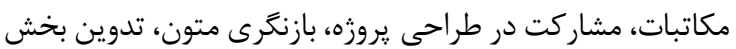

$$
\begin{aligned}
& \text { نتايج و بحث و نعارش مقاله · r درصد. }
\end{aligned}
$$

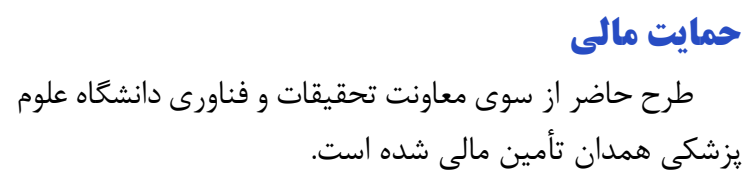

\section{REFERENCES}

1. Stratton I, Kohner E, Aldington S, Turner R, Holman R, Manley S, et al. UKPDS 50: risk factors for incidence and progression of retinopathy in type II diabetes over 6 years from diagnosis. Diabetologia. 2001;44(2):156-63. PMID: 11270671 DOI: $10.1007 / \mathrm{s} 001250051594$

2. Antwi-Bafour S, Hammond S, Adjei JK, Kyeremeh R, Martin-Odoom A, Ekem I. A case-control study of prevalence of anemia among patients with type 2 diabetes. $J$ Med Case Rep. 2016;10(1):110. PMID: 27142617 DOI: 10.1186/s13256-016-0889-4

3. Gedebjerg A, Almdal TP, Berencsi K, Rungby J, Nielsen JS, Witte DR, et al. Prevalence of micro-and macrovascular diabetes complications at time of type 2 diabetes diagnosis and associated clinical characteristics: a cross-sectional baseline study of 6958 patients in the Danish DD2 cohort. $J$ Diabetes Complications. 2018;32(1):34-40. PMID: 29107454 DOI: 10.1016/j.jdiacomp.2017.09.010

4. Rees G, Xie J, Fenwick EK, Sturrock BA, Finger R, Rogers SL, et al. Association between diabetes-related eye complications and symptoms of anxiety and depression. JAMA Ophthalmol. 2016;134(9):1007-14. PMID: 27387297 DOI: 10.1001/jamaophthalmol.2016.2213

5. Kobrin Klein BE. Overview of epidemiologic studies of diabetic retinopathy. Ophthalmic Epidemiol. 2007;14(4):17983. PMID: 17896294 DOI: $10.1080 / 09286580701396720$

6. Romero-Aroca P. Managing diabetic macular edema: the leading cause of diabetes blindness. World J Diabetes. 2011;2(6):98-104. PMID: 21860693 DOI: 10.4239/wjd. v2.i6.98

7. Lee R, Wong TY, Sabanayagam C. Epidemiology of diabetic retinopathy, diabetic macular edema and related vision loss. Eye Vis (Lond). 2015;2:17. PMID: 26605370 DOI: 10.1186/s40662-015-0026-2

8. Lamoureux EL, Tai ES, Thumboo J, Kawasaki R, Saw SM, Mitchell $\mathrm{P}$, et al. Impact of diabetic retinopathy on visionspecific function. Ophthalmology. 2010;117(4):757-65. PMID: 20122736 DOI: 10.1016/j.ophtha.2009.09.035

9. Mohammadnezhad R, Azmodeh M, Tabatabaee S, Hosseini Nasab S. Comparison of active memory, inhibition of response and dimensions of nature and character in patients with type 2 diabetic retinopathy and normal counterparts in Tabriz. Armaghane Danesh. 2020;25(2):368-80. [Persian]

10. Noroozinejad GH, Boustani H, Neamat PS, Behrouzian F. Comparison of depression among diabetic and non-diabetic patients. Jundishapur Sci Med J. 2006;5(1):385-91

11. Grandinetti A, Kaholokula JK, Crabbe KM, Kenui CK, Chen R, Chang HK. Relationship between depressive symptoms and diabetes among native Hawaiians. Psychoneuroendocrinology. 2000;25(3):239-46. PMID: 10737695 DOI: 10.1016/s0306-4530(99)00047-5

12. Bădescu S, Tătaru C, Kobylinska L, Georgescu E, Zahiu D,

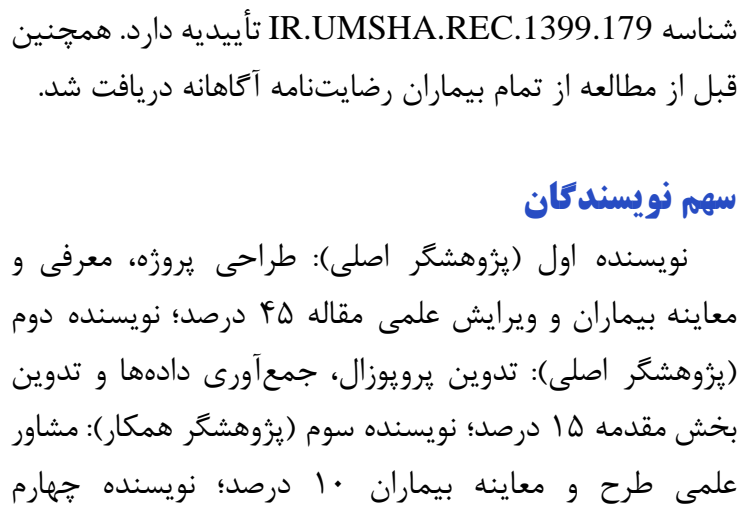

Zăgrean A, et al. The association between diabetes mellitus and depression. J Med Life. 2016;9(2):120-5. PMID: 27453739

13. Chen X, Lu L. Depression in diabetic retinopathy: a review and recommendation for psychiatric management. Psychosomatics. 2016;57(5):465-71. PMID: 27380941 DOI: 10.1016/j.psym.2016.04.003

14. Salinero-Fort MA, Gómez-Campelo P, San Andrés-Rebollo FJ, Cárdenas-Valladolid J, Abánades-Herranz JC, Carrillo de Santa Pau E, et al. Prevalence of depression in patients with type 2 diabetes mellitus in Spain (the DIADEMA Study): results from the MADIABETES cohort. BMJ Open. 2018;8(9):e020768. PMID: 30249627 DOI: 10.1136/ bmjopen-2017-020768

15. Olson D, Le P, Vu T, Van Buren E, Lin FC, Zhang A. Association between anxiety, depression, and severity of diabetic retinopathy. Invest Ophthalmol Vis Sci. 2020; 61(7):3836. DOI: 10.1167/iovs. 18-26108

16. Ahmed M, Gad M, Al-Adlany M. Cognitive impairment and depression in patients with diabetic retinopathy. Egypt $J$ Psychiatry. 2020;41(1):1. DOI: 10.4103/ejpsy.ejpsy $13 \quad 19$

17. Bao Y, Cope S, Gaddis M, Drees B. Prevalence and predictors of depression in patients with diabetic retinopathy in a nationally representative sample. Invest Ophthalmol Vis Sci. 2020;61(7):1902.

18. Abu Ameerh MA, Hamad GI. The prevalence of depressive symptoms and related risk factors among diabetic patients with retinopathy attending the Jordan University Hospital. Eur J Ophthalmol. 2020;22:1120672120912691. PMID: 32202144 DOI: $10.1177 / 1120672120912691$

19. Urrutia-Aliano D, Segura ER. Depressive symptoms and type 2 diabetes mellitus in outpatients of an Armed Forces hospital in Lima, Peru, 2012: a cross-sectional study. Medwave. 2016;16(3):e6435. PMID: 27144926 DOI: $10.5867 / \mathrm{med}$ wave.2016.03.6435

20. Hirai FE, Tielsch JM, Klein BE, Klein R. Relationship between retinopathy severity, visual impairment and depression in persons with long-term type1 diabetes. Ophthalmic Epidemiol. 2012;19(4):196-203. PMID: 22775274 DOI: $10.3109 / 09286586.2012 .692006$

21. Larijani B, Bayat M, Gorgani M, Bandarian F, Akhondzade $\mathrm{S}$, Sadjadi S. Association between depression and diabetes. German J Psychiatry. 2004;7(3):62-5.

22. Roy MS, Roy A, Affouf M. Depression is a risk factor for poor glycemic control and retinopathy in African-Americans with type 1 diabetes. Psychosom Med. 2007;69(6):537-42. PMID: 17634567 DOI: 10.1097/PSY.0b013e3180df84e2

23. Sieu N, Katon W, Lin EH, Russo J, Ludman E, Ciechanowski P. Depression and incident diabetic retinopathy: a prospective cohort study. Gen Hosp Psychiatry. 2011;33(5):429-35. PMID: 21762993 DOI: 10.1016/j.genhosppsych.2011.05.021 